\title{
Comparison of Microbial Diversity and Composition in the Jejunum and Colon of Alcohol-Dependent Rats ${ }^{[\mathrm{S}}$
}

\author{
Yang Fan $^{1,2}$, Zhao Ya-E ${ }^{1 *}$, Wei Ji-dong ${ }^{2}$, Lu Yu-fan', Zhang Ying ${ }^{2}$, Sun Ya-lun ${ }^{2}$, Ma Meng-Yu', and \\ Zhang Rui-ling ${ }^{3}$ \\ ${ }^{1}$ Department of Pathogen Biology and Immunology, School of Basic Medical Science, Xi'an Jiaotong University, Xi'an 710061, P.R. China \\ ${ }^{2}$ Department of Microbiology, School of Basic Medical Science, Xinxiang Medical University, Xinxiang 453003, P.R. China \\ ${ }^{3}$ The Second Affiliated Hospital, Xinxiang Medical University, Xinxiang 453000, P.R. China
}

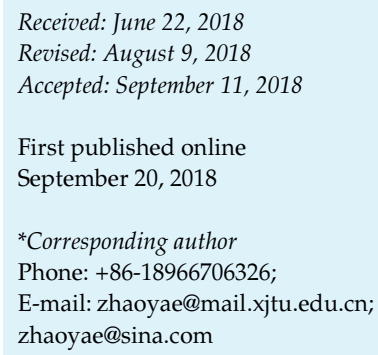

$S$ upplementary data for this paper are available on-line only at http://jmb.or.kr.

pISSN 1017-7825, eISSN 1738-8872

Copyright(C 2018 by

The Korean Society for Microbiology and Biotechnology

\begin{abstract}
Alcohol dependence is a global public health problem, yet the mechanisms of alcohol dependence are incompletely understood. The traditional view has been that ethanol alters various neurotransmitters and their receptors in the brain and causes the addiction. However, an increasing amount of experimental evidence suggests that gut microbiota also influence brain functions via gut-to-brain interactions, and may therefore induce the development of alcohol use disorders. In this study, a rat model of alcohol dependence and withdrawal was employed, the gut microbiota composition was analyzed by high-throughput 16S rRNA gene sequencing, and the metagenome function was predicted by PICRUSt software. The results suggested that chronic alcohol consumption did not significantly alter the diversity and richness of gut microbiota in the jejunum and colon, but rather markedly changed the microbiota composition structure in the colon. The phyla Bacteroidetes and eight genera including Bacteroidales S24-7, Ruminococcaceae, Parabacteroides, Butyricimonas, et al were drastically increased, however the genus Lactobacillus and gauvreauii in the colon were significantly decreased in the alcohol dependence group compared with the withdrawal and control groups. The microbial functional prediction analysis revealed that the proportions of amino acid metabolism, polyketide sugar unit biosynthesis and peroxisome were significantly increased in the AD group. This study demonstrated that chronic alcohol consumption has a dramatic effect on the microbiota composition structure in the colon but few effects on the jejunum. Inducement of colonic microbiota dysbiosis due to alcohol abuse seems to be a factor of alcohol dependence, which suggests that modulating colonic microbiota composition might be a potentially new target for treating alcohol addiction.
\end{abstract}

Keywords: Alcohol dependence, gut microbiota, dysbiosis, jejunum, colon

\section{Introduction}

Alcohol, as a common addictive substance, has long been widely consumed worldwide. Long-term alcohol consumption induces alcohol use disorders (AUDs) including alcohol abuse and dependence, which can be identified by alcohol seeking and craving, and alcohol withdrawal symptoms such as anxiety, restlessness, agitation, tremor, seizures, as well as cognitive dysfunction [1, 2]. Alcohol dependence (AD) brings about not only a negative effect on health, but also criminality and hazards to others while imposing an extremely high burden on society [3,4]. Hence, treating alcohol addiction is certainly an important challenge for the medical community.

Many risk factors may contribute to the development of alcohol dependence including neurobiology [5], environment [6] and psychosocial conditions [7]. However, the formation mechanisms of alcohol dependence have not been fully elucidated to date. The previous studies about the causes of alcohol dependence have mainly focused on 
the effect of alcohol consumption on neuronal functions in the brain. The traditional viewpoint held that ethanol, through the blood-brain barrier, directly or indirectly excites the Ventral Tegmental Area (VTA), increasing the release of some neurotransmitters including dopamine, serotonin, glutamate and $\gamma$-aminobutyric acid (GABA), and causing a rewarding effect, which is a pathophysiological foundation of alcohol dependence [8, 9]. However, the drugs used to treat alcoholism target the neurotransmitter systems and have displayed a limited effect, which suggests that there are other possible peripheral biological processes inducing alcohol dependence [10,11]. Recently, the "brain-gut-microbiota axis" hypothesis has put forth that the gut bacteria may influence brain functions and behavior through many pathways [12, 13]. It is widely accepted that the gut microbiota has important physiological functions and is indispensable for animals and humans alike. It has been proved that intestinal microbiota dysbiosis can cause a variety of somatic diseases including type 2 diabetes [14], allergy [15], inflammatory bowel diseases [16] and obesity [17]. Many documents have also stated that gut microbiota dysbiosis may contribute to some psychiatric disorders such as depression, autism and substance addictions [18-21]. But data about the effects of alcohol dependence on gut microbiota dysbiosis are limited.

Numerous factors are involved in modifying the microbial composition of the gut, including diet, antibiotic use and genetics [22, 23]. Some data also suggest that alcohol and the products of its degradation can strongly disturb the gut microbiota [24, 25]. Bishehsari et al reviewed that chronic alcohol consumption increased gram-negative bacterial overgrowth in the intestine, raised gut permeability and enhanced the plasma levels of gut-derived bacterial products including peptidoglycans and lipopolysaccharides, which contribute to alcoholic hepatitis and can cause injury to other organs [26]. Another study observed that the abundance of Proteobacteria and Actinomycetes increased, but that the normal symbiosis bacteria were reduced after a 3 -wk period of alcohol consumption in rats [27]. Although there also have been some studies done on the gut microbiota regarding alcohol dependence and alcoholism, the samples in these studies were from feces instead of the gastrointestinal tract. Moreover, fecal samples are easily, frequently, and continuously collected, and often act as a substitute for microbial communities in the gut. However, that the fecal microbiota represents all the microbial communities in the gastrointestinal tract is inapposite, and furthermore, the composition of microbiota communities changes with the spatial location within the gut [28]. Some studies in other animal models have demonstrated that microbial communities obviously differ between the gastrointestinal tract and feces as well as between different locations in the gastrointestinal tract [29]. To our knowledge, there has been no literature focusing on gut microbiota variation in different intestinal locations in subjects with alcohol dependence syndrome. Thus, to illuminate further the relationship between gut microbial communities and alcohol dependence syndrome, it is important to reveal the spatial variation in microbiota across the gastrointestinal tract.

In the present study, our aims were to characterize the microbial diversity and composition in the jejunum and colon, and compare the effects of alcohol dependence and alcohol withdrawl on the microbiota of the foregut and hindgut. This study provides stronger evidence for further research on microbial communities and their function in alcohol dependence syndrome.

\section{Materials and Methods}

\section{Animals}

Adult male Wistar rats weighing 190-210 g at the beginning of the experiments were used in this study. They were purchased from Charles River Laboratories, Beijing, China, with the permission of SCXK (Jing) 2016-0011 (No. 11400700259454). The rats were placed in a quiet room with temperature at $\left(21 \pm 2^{\circ} \mathrm{C}\right)$ and humidity of $(50 \pm 5 \%)$, in which $12-12 \mathrm{~h} \mathrm{light-dark} \mathrm{cycle} \mathrm{was}$ maintained (08:00-20:00h light), and the rats were allowed to acclimate to the environment for one week prior to experiment. All animal experimental procedures were approved by the Animal Care and Use Committee of Xinxiang Medical University, China (permission number: AE-2014-09/03).

\section{Alcohol-Dependent Rat Models}

The alcohol dependence models were established as described in documents [30]. Briefly, 40 rats were randomly assigned to control and AD groups. Rats were housed in cages $(460 * 300 * 215 \mathrm{~mm})$, and each group had 4 cages with 5 rats per cage. In AD groups, ethanol was administered in drinking water at gradually increasing concentrations from $1 \%$ to $6 \%(\mathrm{v} / \mathrm{v})$ for the first week, and following this, $6 \%$ alcohol was continued during a total of 30 days. Control group rats received tap water only. All liquid was prepared daily and given to the rats at the same time (a.m. 9:00). The weights of the rats were recorded every week, and the daily drinking water (containing alcohol) was measured every day. The alcohol intake of individual animals was expressed as the average value of each cage. At the end of the 30 days, ethanol was withdrawn from the drinking water by providing only tap water until the end of experiments in the AD group. 
Withdrawal syndrome was observed for 5 min at 1, 3, 5, 9 days of the ethanol-withdrawal period. The behavioral parameters were assessed according those previously described [30], with main prameters including: locomotor activity, body posture, gait, agitation, tail stiffness, tremor and stereotyped behavior. Locomotor activity was measured by open field test and the major stereotype behaviors include head weaving, grooming, gnawing, sniffing, and chewing during the ethanol withdrawal. At the 5-day and 14day points of the withdrawal testing, rats were exposed to an audiogenic stimulus $(100 \mathrm{~dB})$ for $1 \mathrm{~min}$ to assessed audiogenic seizure.

\section{Sampling and DNA Extraction}

On the day before ethanol withdrawl (T0) and 14 days after detoxification (T1), rats were randomly selected (6 per group) and euthanized. The jejunum and colon contents were collected into sterile eppendorf tubes and stored at $-80^{\circ} \mathrm{C}$. Total genomic DNA in the jejunum and colonic contents were extracted from $0.3 \mathrm{~g}$ of samples with a Quick Gel Extraction Kit (Qiagen, Germany) as per the manufacturer's instructions. The quantity and concentration of genomic DNA were measured using a ThermoNanoDrop 2000C (Thermo Fisher Scientific, USA).

16S rRNA Gene Amplification and High-Throughput Sequencing

The $16 \mathrm{~S}$ rDNA was amplified using primers 338F and 806R which covered the hyper-variant region $\mathrm{V} 3-\mathrm{V} 4$ regions of bacteria by PCR. The forward primer is 338F $5^{\prime}$-ACTCCTACGGG AGG CAGCA-3' and reverse primer is $806 \mathrm{R}$ 5'-GGACTACHVGGGTWT CTAAT-3'. The 6 bp-long, sample-specific barcodes were designed in the two primers to avoid homopolymers. A detailed list containing the sequences of the barcodes can be found in Table S1. The PCR reactions were performed according to the following volume: $20 \mu \mathrm{l}$ reaction mixtures containing $10 \mathrm{ng}$ DNA template, $0.5 \mu \mathrm{l}$ each primers $(5 \mu \mathrm{M}), 0.5 \mu \mathrm{l}$ FastPfu polymerase, $2.5 \mathrm{mM}$

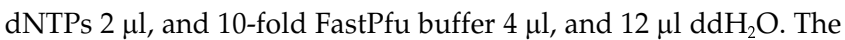
following program of $\mathrm{PCR}$ reactions was conducted: $3 \mathrm{~min}$ of denaturation at $95^{\circ} \mathrm{C}, 27$ cycles of $30 \mathrm{sec}$ at $95^{\circ} \mathrm{C}, 30 \mathrm{sec}$ for annealing at $55^{\circ} \mathrm{C}$, and $45 \mathrm{sec}$ for elongation at $72^{\circ} \mathrm{C}$, and a final extension at $72^{\circ} \mathrm{C}$ for $10 \mathrm{~min}$. The resulted PCR products were checked on a $2 \%$ agarose gel and further purified using the AxyPrep DNA Gel Extraction Kit (Axygen Biosciences, Union City, USA) according to the manufacturer's protocols. Purified amplicons were pooled in equimolar and paired-end sequenced $(2 \times 300)$ on an Illumina MiSeq platform (Illumina, USA) according to the standard protocols by Majorbio Bio-Pharm Technology Co., Ltd. (China).

\section{Bioinformatic Analysis}

The raw reads of all samples in this study have been submitted to the NCBI Sequence Read Archive Database under accession number SRP139712. In order to obtain the qualified sequences, the following criteria were used: (i) the reads were truncated at any site receiving an average quality score $<20$ over a 50 bp sliding window. (ii) Primers were exactly matched allowing 2 nucleotide mismatching, and reads containing ambiguous bases were removed. (iii) Sequences whose overlap was longer than $10 \mathrm{bp}$ were merged according to their overlap sequence. Operational taxonomic units (OTUs) were clustered with $97 \%$ similarity cutoff using UPARSE (version $7.1 \mathrm{http} / /$ /drive5.com/uparse/) and chimeric sequences were identified and removed using UCHIME. The taxonomy of each 16S rRNA gene sequence was analyzed by RDP Classifier algorithm (http://rdp.cme.msu.edu/) against the Silva (128) 16 S rRNA database using confidence threshold of 70\%. The alpha-diversity indices including Shannon, Chao, ACE and Simpson were calculated for estimating microbiota diversity and abundance in each sample. The beta-diversity indices (PCoA) were calculated to show the composition of the gut microbiota communities in the different gut segment samples, and analysis of molecular variance (AMOVA) was performed to compare the difference between jejunum and colon. The LEfSe (linear discriminant analysis [LDA] coupled with effect size measurements) analysis was conducted to calculate the biomarkers between the groups. Correlation analysis between alcohol withdrawl syndrome and genera with significant differences and two-tailed test were performed using Statistical Package for the Social Sciences (SPSS, version 17.0) based on Pearson correlation coefficient.

\section{Microbial Functional Predictions and Statistical Analysis}

The microbial metagenomic functional prediction was performed with PICRUSt (phylogenetic investigation of communities by reconstruction of unobserved states) software. OTUs were normalized by copy numbers, and the gene categories were predicted by KEGG (Kyoto Encyclopedia of Genes and Genomes) orthology groups (KOs) and KEGG pathways of bacterial communities. The functional differences among groups were compared through the software STAMP. Sequence and OTU richness and diversity indices were tested via one-way ANOVA. The Wilcoxon ranksum test was used to compare controls, AD and AW groups. Statistical analyses were performed using SPSS version 20.0 for Windows. Heat-map diagrams and other plotting were carried out in the R environment.

\section{Results}

\section{Establishment of the Alcohol-Dependent Models of Rats}

Alcohol-dependent models were established by longterm freedom drinking of $6 \%$ ethanol liquor. The results showed the average daily liquor consumption of the rats was $110.83 \mathrm{ml} / \mathrm{kg}$, which was equivalent to $5.25 \mathrm{~g} / \mathrm{kg}$ of pure alcohol intake. The locomotor activity and behavioral change signs of ethanol withdrawal syndrome were observed by the double-blind method. The results showed that there was significant difference in locomotor activity and total ethanol withdrawal scores between the alcohol dependence group and the control group ( $p<0.05$, Fig. 1). 


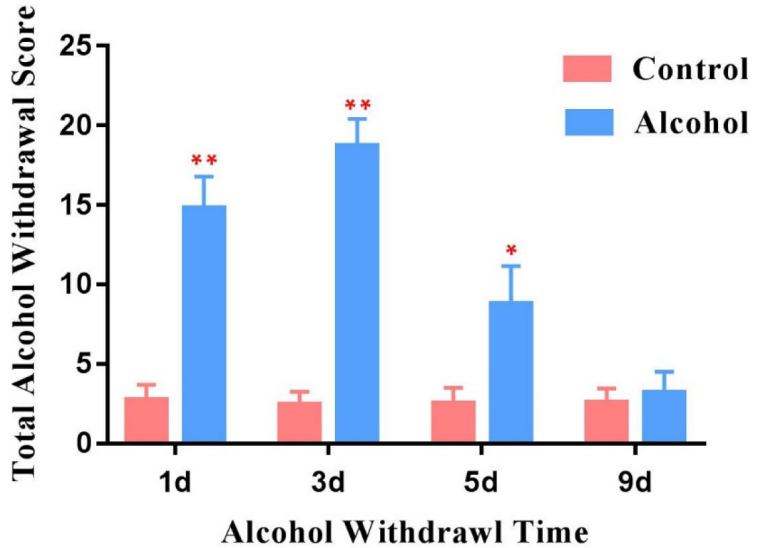

Fig. 1. Total alcohol withdrawl scores at different periods of ethanol withdrawal.

The values are express as the mean $\pm \operatorname{SD}(n=12),{ }^{*} p<0.05,{ }^{* *} p<0.01$, Mann-Whitney U-tests, significantly different between ethanol dependent group vs control group.

The rats in the ethanol dependent group displayed locomotor hyperactivity after withdrawal ethanol, and the highest ethanol withdrawal scores were observed at the third day of ethanol withdrawal. When compared with the control group, the total ethanol withdrawal scores in the
$\mathrm{AD}$ group showed no distinct difference after withdrawal of ethanol for 9 days. With a 100Bd audiogenic stimulus for one minute, the audiogenic seizures appeared with $75 \%$ $(9 / 12)$ in the $\mathrm{AD}$ group and $8.30 \%(1 / 12)$ in the control group after withdrawing alcohol for 5 days. Meanwhile, the incidence of audiogenic seizures was $33.33 \%(4 / 12)$ in the AD group and $8.30 \%(1 / 12)$ in the control group after withdrawal of alcohol for 14 days. All these results indicated that daily ethanol consumption could produce physical dependence symptoms in rats.

\section{Summary of High-Throughput Sequencing}

In total 1,873,879 reads were obtained after quality control and chimera filtering. On average, 44,940 and 59,164 sequences per sample were obtained from the jejunum and colon contents, respectively, with an average length of $440 \mathrm{bp}$ (Table S2). A total of 3,856 OTUs (Operational Taxonomic Units) were identified with the criterion of $97 \%$ sequence similarity at the species level, on average, 1,878 and 1,978 OTUs were assigned to the jejunum and colon contents, respectively (Table S3).

\section{Effect of Alcohol on Richness and Diversity of Gut Microbial Communities}

The high-throughput sequencing results showed that a

Table 1. Richness and diversity indices estimation of the jejunum and colonic contents in rats.

\begin{tabular}{|c|c|c|c|c|}
\hline \multirow{2}{*}{ Groups } & \multicolumn{2}{|c|}{ Richness indices } & \multicolumn{2}{|c|}{ Diversity indices } \\
\hline & Chao1 & Ace & Shannon & Simpson \\
\hline Jejunum $(n=18)$ & $266.4 \pm 175.22$ & $304.67 \pm 177.07$ & $2.18 \pm 0.72$ & $0.22 \pm 0.08$ \\
\hline Colon $(n=18)$ & $522.1 \pm 27.38$ & $515.77 \pm 24.88$ & $3.84 \pm 0.44$ & $0.07 \pm 0.03$ \\
\hline$p$-value & $3.254 \mathrm{e}-7^{*}$ & $1.028 \mathrm{e}-5^{*}$ & $4.494 \mathrm{e}-10^{*}$ & $1.22 \mathrm{e}-8^{*}$ \\
\hline \multicolumn{5}{|l|}{ JEJUNUM } \\
\hline Control $(n=6)$ & $183.02 \pm 112.29$ & $218.06 \pm 99.67$ & $2.18 \pm 0.39$ & $0.19 \pm 0.05$ \\
\hline $\mathrm{AD}(n=6)$ & $258.8 \pm 124.98$ & $277.95 \pm 123.62$ & $2.19 \pm 0.31$ & $0.21 \pm 0.04$ \\
\hline $\mathrm{AW}(n=6)$ & $357.37 \pm 240.45$ & $418.01 \pm 236.88$ & $2.18 \pm 1.23$ & $0.26 \pm 0.13$ \\
\hline$p$-value (AD vs Control) & 0.30 & 0.38 & 0.98 & 0.61 \\
\hline$p$-value (AW vs Control) & 0.14 & 0.09 & 0.99 & 0.26 \\
\hline$p$-value (AD vs AW) & 0.39 & 0.23 & 0.99 & 0.35 \\
\hline \multicolumn{5}{|l|}{ COLON } \\
\hline Control $(n=6)$ & $517.3 \pm 15.07$ & $511.17 \pm 16.53$ & $3.82 \pm 0.31$ & $0.06 \pm 0.02$ \\
\hline $\mathrm{AD}(n=6)$ & $525.61 \pm 33.29$ & $520.79 \pm 25.93$ & $3.82 \pm 0.51$ & $0.07 \pm 0.04$ \\
\hline $\mathrm{AW}(n=6)$ & $512.71 \pm 19.82$ & $504.78 \pm 16.62$ & $3.76 \pm 0.46$ & $0.07 \pm 0.04$ \\
\hline$p$-value (AD vs Control) & 0.59 & 0.46 & 0.99 & 0.49 \\
\hline$p$-value (AW vs Control) & 0.66 & 0.52 & 0.83 & 0.51 \\
\hline$p$-value (AD vs AW) & 0.43 & 0.23 & 0.85 & 0.99 \\
\hline
\end{tabular}

*Indicates a significance between jejunum and colon, AD: alcohol-dependent; AW: alcohol-withdrawal 
total of the 808,927 sequences were obtained from the jejunum samples, the smallest number being 33,172 in the $\mathrm{AD}$ group, and the largest number being 64,888 in the control group. There were 1,064,952 sequences in the colon contents, and the responding number is 42,022 and 77,498 in the AW group and control group, respectively (Table S2). But there were no significant differences between the three treatment groups $(p>0.05)$. To determine if there were differences in the richness and diversity of microbial populations within the jejunum and colon contents, the richness indices (Ace and Chao 1) and diversity indices (Shannon and Simpson) were compared between different treatment groups. We combined samples according to gut location for analyzing microbial composition structure, with the alpha diversity showing significantly difference between the jejunum and colon samples $(p<0.001$, Table 1$)$, which indicated that gut location had a significant impact on the microbial composition structure in the rats. Next, we separately compared microbiota composition in the same gut location between the different treatment groups. Oneway analysis of variance (ANOVA) showed no significant differences between the three groups in microbial richness and diversity in the jejunum or colon contents $(p>0.05$, Table 1), which suggested that alcohol consumption and alcohol withdrawal did not distinctly impact on microbial diversity and richness of the jejunum and colon in rats.

To further reveal the impact of alcohol dependence and withdrawal on the microbiota structure, the PCoA were performed based on unweighted (presence/absence information) Unifrac distances. The PCoA results showed that among different samples clustered primarily by intestinal segment, all the foregut samples (jejunum, green) differed from the hindgut samples (colon, red) (Fig. 2A), which indicated the microbiota composition structure was distinctly different between the jejunum and colon. In the jejunum samples, all three groups were not obviously clustered separately based on the principal coordinate (Fig. 2C), which indicated chronic alcohol consumption and alcohol withdrawal did not significantly alter the microbiota composition structure of the jejunum. However, in the colonic contents, both $\mathrm{AW}$ and control groups had similar microbial community structures that differed from those of the AD group (Fig. 2B), indicating ethanol consumption had distinctly impacted the microbial structure of the colon. We further analyzed the relationships in intestinal microbial community structure between groups by using ANOSIM based on Bray-Curtis distance at the OTU level. The results showed that the community compositions bore no significant differences within groups
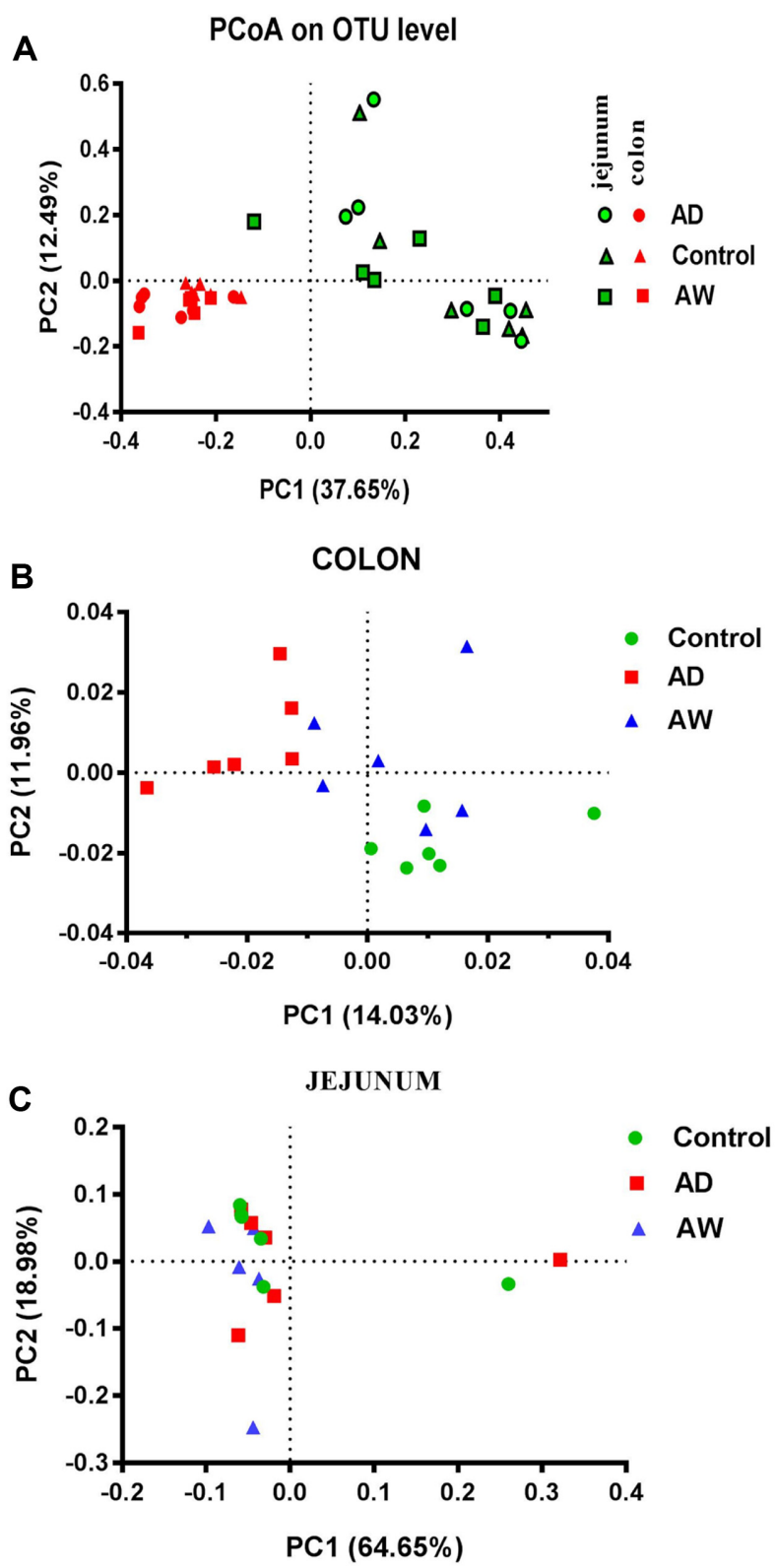

Fig. 2. Principal coordinate analysis (PCoA) by unweighted UniFrac distance.

(A) All the samples including jejumun and colon contents; (B) colon samples; (C) jejunum samples. The microbiota construct of foregut samples differed from that of hindgut samples (A); the microbiota construct of the $\mathrm{AD}$ group was significant different from that of the control group in the colon (B); no significant differences between the three groups in the jejunum (C). AD: alcohol-dependent; $A W$ : alcohol-withdrawl.

or among groups $\left(R^{2}=0.074, p>0.05\right.$, Tables 2,3$)$ in the jejunum samples. But in colonic samples, the microbial community structure of the $\mathrm{AD}$ group was significantly 
Table 2. Pseudo F table of ANOSIM analysis based on Bray-Curtis dissimilarities on OTU level ( $n=18$ per group).

\begin{tabular}{lcccccc}
\hline Source of variance & Degrees of freedom & Sum of squares & Mean square & $\mathrm{F}$ & $\mathrm{R}^{2}$ & $p$. value \\
\hline Jejunum groups & 2 & 0.223 & 0.111 & 0.596 & 0.074 & 0.837 \\
Colon groups & 2 & 0.438 & 0.219 & 1.784 & 0.192 & $0.037^{*}$ \\
\hline
\end{tabular}

*Indicates significant difference within groups

different from that of the control group $\left(\mathrm{R}^{2}=0.192, p<0.05\right.$, $\mathrm{R}^{2}=0.240, p<0.01$, Tables 2,3$)$. On the other hand, the gut microbial community compositions revealed no significant differences between the AD group and AW group $\left(R^{2}=0.115, p=0.234\right.$, Tables 3$)$. These results suggest that chronic alcohol consumption has a markedly greater effect on the gut microbiota structure of the colon than that of the jejunum in rats.

\section{Effect of Alcohol Dependence and Withdrawal on Bacterial Composition}

At the phyla level, after filtering the relative abundance lower than $0.02 \%$ in all groups, a total of 12 and 8 phyla were identified in the jejunum and colon microbiota, respectively (Tables S4, S5). Firmicutes, Proteobacteria and Actinobacteria were the three dominant microbial phyla (the relative abundance $>1 \%$ ) in the jejunum contents. Although the abundance of Firmicutes was decreased and Proteobacteria was increased in the AD group compared with the control group, one-way ANOVA showed no significant difference between the two groups ( $p>0.05$, Table S4). In the colonic microbiota, Firmicutes and Bacteroidetes were the dominant phyla, occupying more than $98.73 \%$ of the total sequences (Table S5). By comparison with the control group, we found the AD group the decreased abundance of Firmicutes with $88.97 \%(p>0.05)$, while showing dramatically increased proportions of Bacteroidetes at $9.83 \% \quad(p<0.01)$ and Proteobacteria at $0.16 \%(p>0.05)$, respectively (Table S5). After alcohol withdrawal, there was a tendency toward further exacerbation of the changes induced by $\mathrm{AD}$ in the Proteobacteria $(p<0.05$,$) and Bacteroidetes ratios (p>0.05)$
(Table S5). When compared with the control group, there were no significant differences in the abundance of other phyla in the AD and AW groups in the colon.

At the class level, after filtering the relative abundance lower than $0.02 \%$ in all groups, a total of 18 and 13 microbial classes were identified from the jejunum and colon microbiota, respectively (Tables S6, S7). In the jejunum, Bacilli, Clostridia and Erysipelotrichia were the most predominant classes, occupying more than $98.73 \%$ of the total sequences (Table S6). All the classes of microbiota in the jejunum contents showed no significant differences between the three groups $(p>0.05)$, which indicated alcohol consumption and withdrawal did not distinctly affect microbial composition in the jejunum. In the colon contents, Clostridia (47.64\%-60.54\%), Bacilli (19.57\%-37.77\%), Bacteroidia $(6.33 \%-11.04 \%)$ and Erysipelotrichia $(5.15 \% \sim 8.41 \%)$ were the dominate classes in these three groups (Table S7). As shown in Table S7, AD treatment numerically increased the relative abundance of Clostridia $(p>0.05)$ and Bacteroidia $(p<0.05)$ while significantly decreasing the relative abundance of Bacilli $(p<0.05)$. Notably, these changes were restored by alcohol-withdrawl treatment for 2 weeks.

To understand what dominant kinds of bacteria were affected by alcohol dependence and withdrawal, we further analyzed the composition of the intestinal microbiota from order to the genus level. The relative abundance lower than $0.05 \%$ in all groups was filtered, and a total of 22 and 12 orders (Tables S8, S9), and 39 and 25 families (Tables S10, S11) were identified in the jejunum and colonic microbiota, respectively. In the jejunum, Lactobacillales, Clostridiales, Burkholderiales and Erysipelotrichales were dominant

Table 3. Pseudo F table of pairwise comparisons ANOSIM based on OTU level ( $n=6$ per group).

\begin{tabular}{|c|c|c|c|c|c|}
\hline Pairwise comparison & Source of variance & Sum of squares & F & $\mathrm{R}^{2}$ & $p$. value \\
\hline AD vs Control & Jejunum groups & 0.132 & 0.796 & 0.073 & 0.489 \\
\hline AW vs Control & Jejunum groups & 0.112 & 0.578 & 0.054 & 0.819 \\
\hline AD vs AW & Jejunum groups & 0.091 & 0.449 & 0.042 & 0.815 \\
\hline AD vs Control & Colon groups & 0.334 & 30171 & 0.240 & $0.001^{*}$ \\
\hline AW vs Control & Colon groups & 0.147 & 1.153 & 0.103 & 0.291 \\
\hline
\end{tabular}

*Indicates significant difference between alcohol dependent group and control group, AD: alcohol-dependent; AW: alcohol-withdrawl 


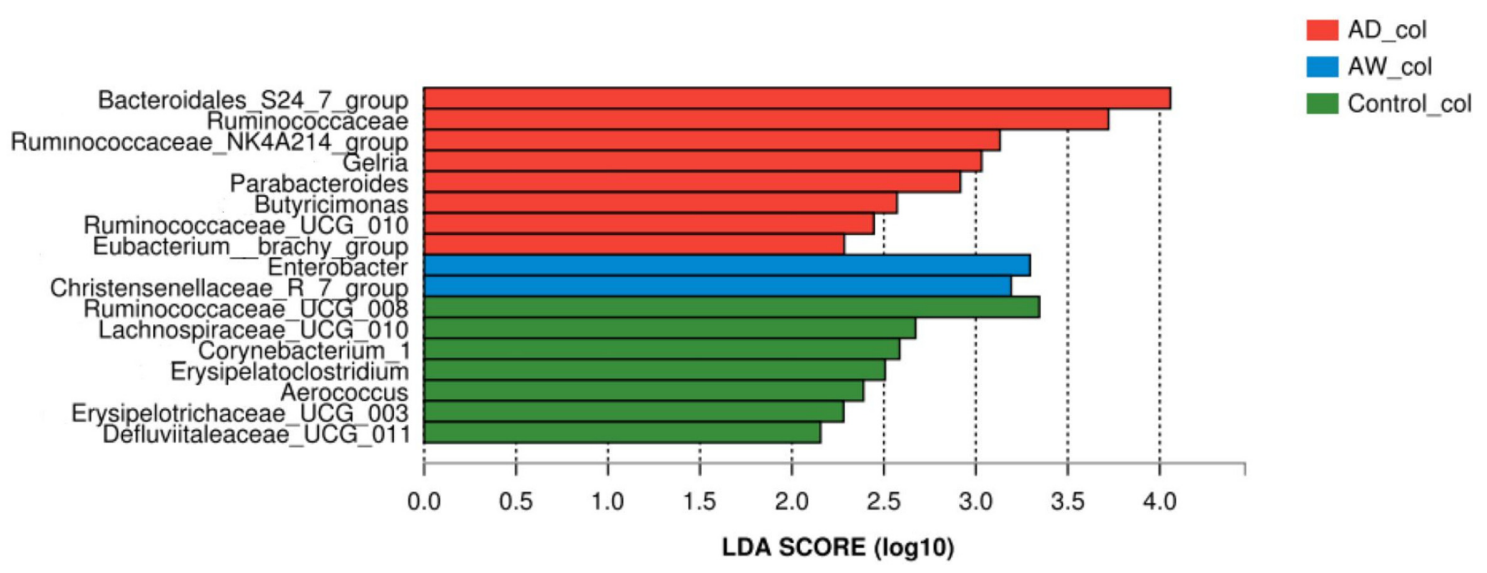

Fig. 3. LEfSe analysis illustrating differentially abundant bacterial genera among samples in colon.

Only those genera that obtain a log LDA score of $>2$ are ultimately considered, while LDA scores can be interpreted as the degree of consistent difference in relative abundance between $\mathrm{AD}, \mathrm{AW}$, and control groups. AD: alcohol-dependent; AW: alcohol-withdrawl.

orders (the relative abundance $>1 \%$ ), and Lactobacillaceae, Erysipelotrichaceae, Peptostreptococcaceae, Streptococcaceae and Oxalobacteraceae were the dominant families (the relative abundance $>1 \%$ ) (Tables S8, S10). There was no significant difference between the three groups at the order and family levels in the jejunum microbes. Clostridiales, Lactobacillales, Bacteroidales and Erysipelotrichales were the four dominant orders, and Lactobacillaceae, Lachnospiraceae, Ruminococcaceae, Peptostreptococcaceae, Erysipelotrichaceae, Rikenellaceae, Prevotellaceae and Bacteroidales-S24-7 were the dominant families (the relative abundance $>1 \%$ ) in colonic microbiota (Tables S9, S11). Interestingly, chronic alcohol consumption obviously increased the abundance of Lactobacillales and decreased the Bacteroidales ratio in the colon $(p<0.05$, Table S9). Five bacterial families including Lactobacillaceae, BacteroidalesS24-7, Bacteroidaceae, Christensenellaceae and Porphyromonadaceae in the AD group were significantly different vs control in the colon $(p<0.05$, Table S11). The abundance of Lactobacillaceae in the AD group was significantly decreased, but the other four families in the AD group were significantly increased compared with control. Instead, alcohol withdrawl treatment restored these changes.

On the genus level, after filtering the abundance $<0.05 \%$ in all groups, a total of 63 and 74 genera were detected in the jejunum and colon, respectively (Tables S12, S13). The most abundant genera (with $>1 \%$ abundance) were Lactobacillus, Romboutsia, Turicibacter and Streptococcus in the jejunum. Similarly, as with the above comparisons in the phyla, class, orders and family, there were no distinct differences in predominant genera between any two groups in the jejunum contents. However, in the colon, the genera numbers of the relative abundance of more than $1 \%$ in the three groups were 15, including Lactobacillus, Romboutsia, Turicibacter, Ruminococcaceae UCG-005 et al. (Table S13). Using the linear discriminant analysis effect size (LEfSe), we identified a large number of taxa that were significantly increased in the $\mathrm{AD}$ group, such as Bacteroidales S24-7, Ruminococcaceae, Gelria, Thermoanaerobacterales and Butyricumonas, et al. (Fig. 3). However, only two genera distinctly decreased in the $\mathrm{AD}$ group, including Lactobacillus and Gauvreauii $(p<0.05$, Fig. S1 and Table S13). Interestingly, in most, alcohol withdrawl treatment can partly restore these microbiota changes induced by alcohol abuse, but the relative abundance of Brachy and Christensenellaceae_R-7 showed opposite changes in the AW group (Figs. 3, S1, and Table S13). Furthermore, correlation analysis between alcohol dependence syndrome and genera showed that Parabacteroides, Butyricimonas, Ruminococcaceae_UCG-010, Bacteroidales_S24-7_group, Gelria, and Ruminococcaceae_ NK4A214_group significantly correlated with alcohol withdrawal syndrome, which indicated that these taxa may play a role in the development of alcohol addictions (Table S14).

Collectively, the above results indicate that chronic alcohol consumption has profound effects on the microbiota compositon structure in the colon but little effect on that of the jejunum.

\section{Microbiota Function Prediction of Colon}

We predicted the functional profiles among the three groups in colonic microbiota by PICRUSt software. KEGG level 1 showed that the category "metabolism" had the 


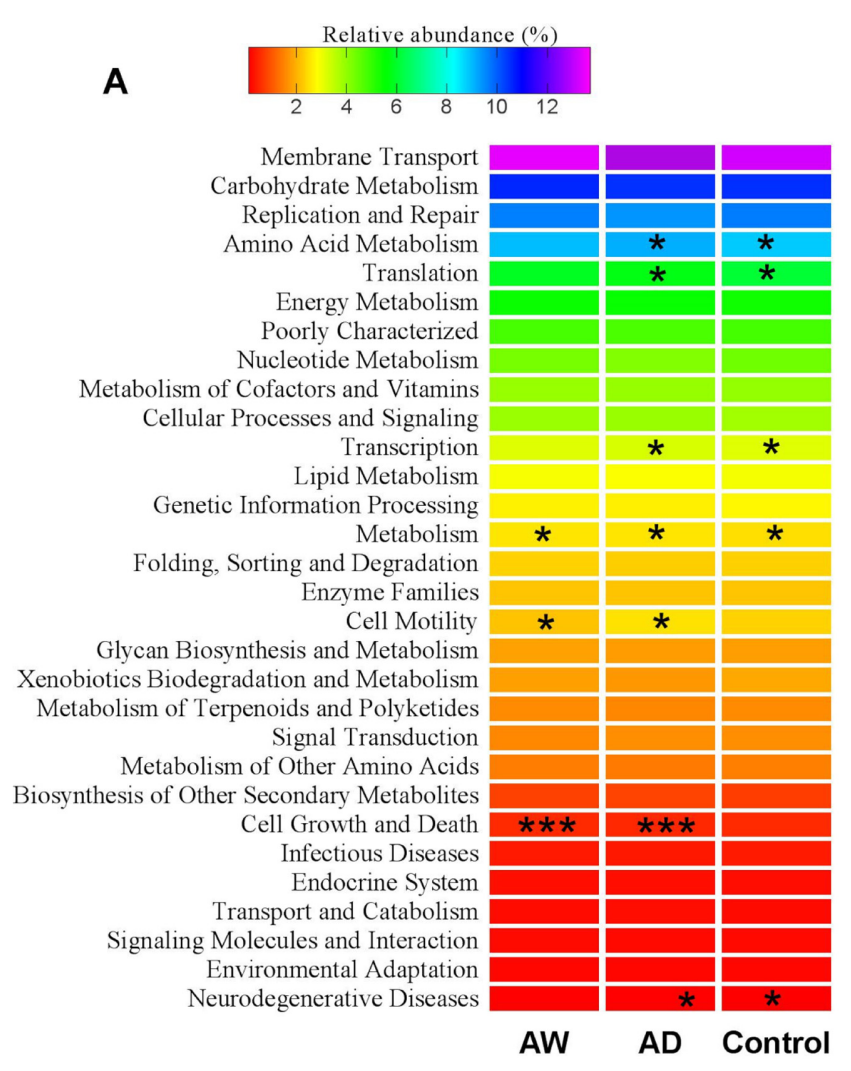

Fig. 4. Predicted microbial function comparison based on KEGG level-2.

The same marker indicates significant difference between two groups, ${ }^{*} p<0.05,{ }^{* * *} p<0.01$, Statistics were conducted by two-sided Welch's t-test. AD: alcohol-dependent; AW: alcohol-withdrawl.

highest relative abundance $(>45 \%)$ among the groups (Fig. S2). At KEGG level 2, a total of 41 gene families were identified in the colon and the relative abundance $(>0.5 \%)$ was present on Fig. 4. Fig. 4 also showed that several majorities of the function genes carried significant difference between groups, including amino acid metabolism, translation, and transcription, et al. Interestingly, the proportion of neurodegenerative diseases was significantly increased in the AD group ( $p<0.05$, vs the control group, Fig. 4A). We further analyzed the metabolic pathways at KEGG level 3. The results showed that $254 \mathrm{KO}$ pathways were identified and 43 dominant $\mathrm{KO}$ pathways (the relative abundance $>0.8 \%$ ) were shown in Fig. 3. Compared with the control group, the proportions of amino acid metabolism, phenylalanine metabolism, polyketide sugar unit biosynthesis and peroxisome were significantly increased and glycosyltransferases, restriction enzyme and chloroalkane and chloroalkene degradation were distinctly decreased in the AD group ( $p<0.05$, Fig. 5A). Instead, after alcohol withdrawal, the relative abundance of peptidases and protein export were greatly increased $(p<0.05)$, while arginine and prolin metabolism, bacterial motility proteins and two-component system were significantly decreased $(p<0.05$, vs AD group, Fig. 5B). Interestingly, alcohol withdrawal restored the changes of most above functional pathways, but it exacerbated the differences in the ratio of amino acid metabolism $(p<0.05$, Fig. 5C). These results implied that alcohol dependence and withdrawl possibly have a dominant effect on amino acid metabolism.

\section{Discussion}

Gut microbiome composition and function are affected by many factors including age, geographical origin, lifestyle and medications. Alcohol is also one of the disruptive agents for gut microbiota. However, the research of alcohol's impacts on the diversity and composition structure of gut microbiota still is in its infancy, particularly compared with other alcohol-induced disease. In the present study, by high-throughput sequencing of $16 \mathrm{~S}$ rRNA genes, we analyzed the impact of chronic alcohol exposure on the microbial community diversity and functions in the jejunum and colon of rats. The results revealed that the chronic alcohol consumption induced quantitative and qualitative changes in the gut microbiota.

To the best our knowledge, there have been limited studies reporting the effects of alcohol on the microbiota in humans [24, 31, 32] and rodents [33, 34]. Most of these studies seem to be relevant for alcohol-associated pathologies such as liver disease [27, 33], and the samples in these studies are mostly collected from feces. However, the samples of this study were collected from jejunum and colon contents, which reflects a more realistic living environment of microbiota. In the present study, the alpha diversity results showed that the gut location was a main important factor of impact on the microbial structure in rats (Table 1), which was also confirmed in other animal models [35]. However, in the same gut location, chronic alcohol consumption and alcohol withdrawal did not obviously alter microbial diversity and richness (Table 1), which was agreed with in a recent report [36], but disagreed with in other reports [33, 34, 37]. The studies in mice and rats find that chronic alcohol treatment can induce bacterial overgrowth in the colon and cecum [33, 34]. Recent studies also demonstrate that excessive alcohol consumption has significant effect on the abundance of enteric bacteria in these alcohol addiction mouse models [36]. To date, we have not checked for the relative 
A

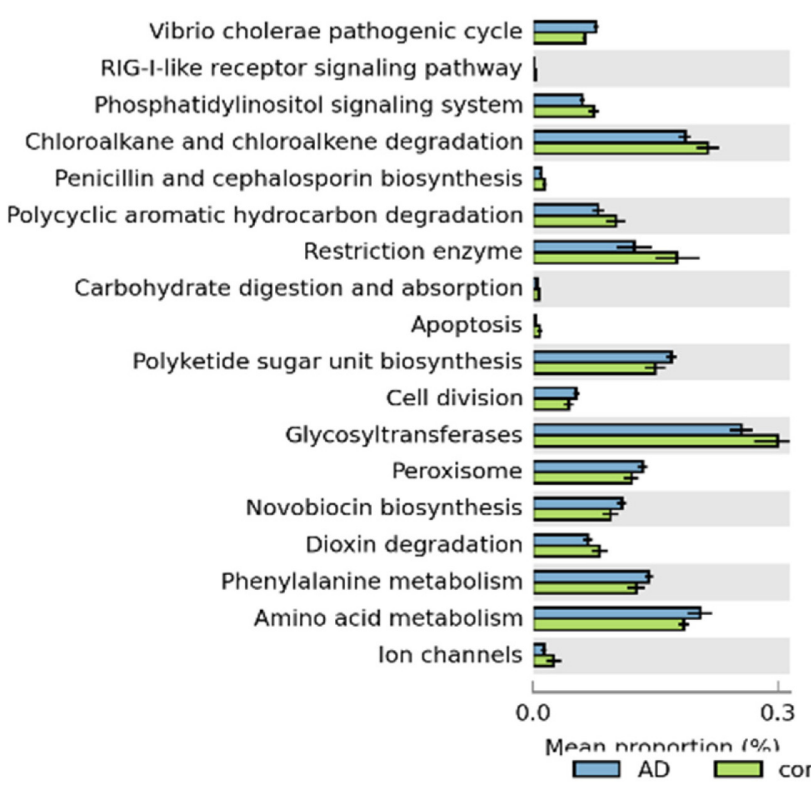

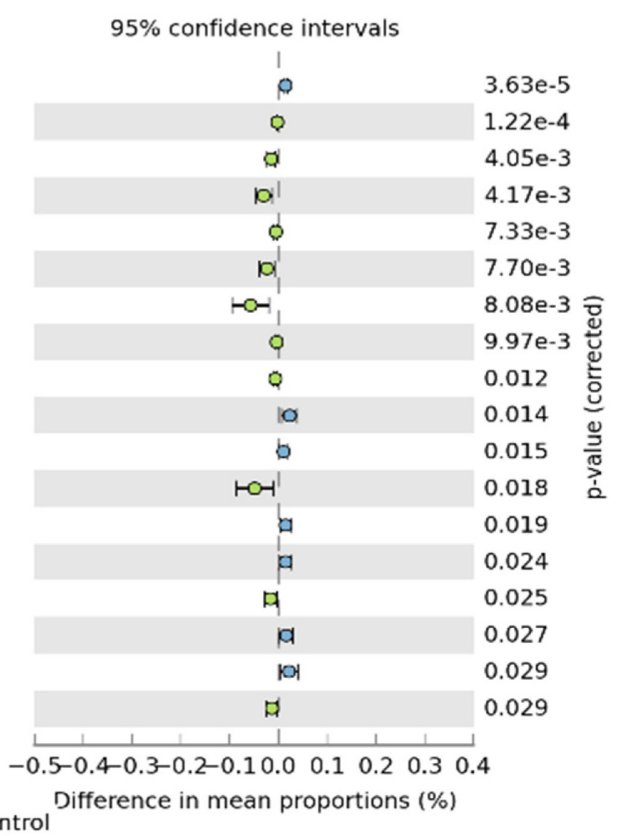

B
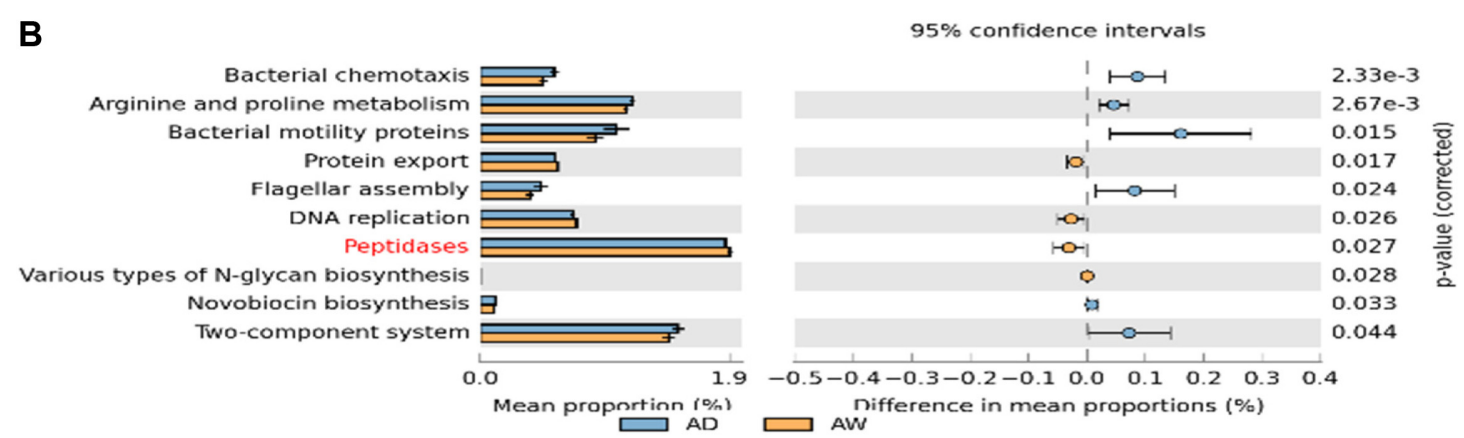

C

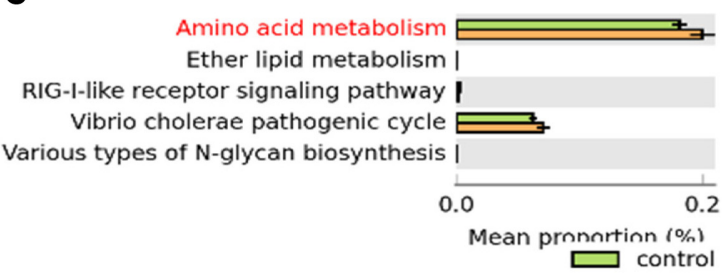

Fig. 5. Predicted microbial function comparison of the colon base on KEGG level-3.

Comparing colonic microbial function between $\mathrm{AD}$ and control (A), AD vs AW (B), AW vs Contol (C). Statistics were conducted by two-sided Welch's t-test and Benjamini-Hochberg FDR correction between two groups, and the P-value of different functions lower than 0.05 were shown. AD: alcohol-dependent; AW: alcohol-withdrawl.

documents about alcohol's effect on the microbiota of the jejunum. In the present study, from the phylum to the genera levels of bacteria, significant differences between the three groups in the jejunum samples were not found. That is to say, chronic alcohol exposure does not distinctly alter the microbiota composition construct of the jejunum. However, others and our studies showed alcohol significantly altered the microbiota composition structure in the larger intestine and feces. These suggested that alcohol metabolite, rather than ethanol itself, is the 
most important factor that appears to alter microbiota composition. Pharmacokinetic studies have shown that $90 \%$ of ingested ethanol is absorbed through the stomach and the proximal small intestine. The ethanol concentrations in the large intestine approximate those in the blood [38, 39]. After being absorbed, ethanol was metabolized through oxidative and non-oxidative pathways, and resulted in acetaldehyde, fatty acid ethyl esters, and phosphatidyl ethanol [40]. These metabolites of ethanol can accumulate in the large intestine via the systemic circulation, and induce microbiota dysbiosis in the large intestine. Nosova et al. [41] demonstrated Lactobacillus GG have a high acetaldehydemetabolizing capacity, but excessive acetaldehyde can inhibit its growth, which identified with our results.

In addition, our discoveries in this paper showed that both alcohol dependence and alcohol withdrawl significantly altered the microbiota composition of the colonic digests (Table 3 and Fig. 2). At the phyla level, Firmicutes and Bacteroidetes were the dominant phyla, and the abundance of Bacteroidetes and Proteobacteria dramatically increased while Firmicutes decreased in the colon after chronic alcohol treatment, which were similarly with other reports. Yan and colleagues investigated the changes in the intestinal microbial community after 3 weeks of alcohol consumption. They observed that relative abundance of Bacteroidetes increased and Firmicutes decreased in alcoholfed mice compared with control mice [34]. Bull-Otterson showed a decline of both Bacteriodetes and Firmicutes phyla in alcohol-fed mice, with an increase in Proteobacteria phyla [27]. In contrast with rodent models, some studies investigated the relationship between alcohol abuse and changes in human gut microbiota showed different results. Leclercq et al. (2014) compared 60 patients with alcohol dependence and 15 healthy subjects to assess the changes of intestinal bacteria, and the results showed no significant differences between the groups of subjects at the phylum level [42]. However, Mullu and colleagues observed that the abundance of Proteobactera and Firmicutes increased and Bacteroidetes decreased in alcohol abuse patients [24]. In all these studies, Gram-negative Proteobacteria phyla increased. Lipopolysaccharides (LPS) are main components of gramnegative bacteria and can provide tonic stimulation of the innate immune system and induce central nervous system inflammation [26]. LeClercq et al have reported elevated blood LPS levels associated with psychological symptoms of alcohol dependence in alcohol-dependent subjects [43].

At the genus level, a drastic increase in relative abundance of eight main genera including Ruminococcaceae (Fig. 3), and a decreased in abundance in the Lactobacillus and
Gauvreauii (Fig. S1) were observed in this study. Previous preclinical study showed that chronic ethanol administration induces in mice a decrease in the level of Ruminococcaceae [27], which was in disagreement with our results. Kirpich et al. (2008) observed a decrease in Lactobacillus in the stool cultures of $\mathrm{AD}$ subjects compared with those of healthy controls, which was similar with our results [44]. Noteworthy, the detected decrease of Lactobacillus in this study is contrary to recently observations for the alcoholic dependence and liver cirrhosis by Dubinkina [45]. Lactobacillus was a reputation of "beneficial" agents in the human gut capable of anti-inflammatory activities, protecting against pathogens. Thus, it is being actively used for treatment of various disorders including Alzheimer's, autism and alcohol liver disease [46, 47]. However, our observations revealed that alcohol consumption reduced the relative abundance of Lactobacillus, indicating that Lactobacillus was a potential agent for treating alcohol disorders.

Based on the predicted PICRUSt of colon bacteria, the present study revealed many pathways related to metabolism at KEGG level 3. Among them, transporters and $A B C$ transporters were the most abundant in our study (Fig. S3), which were important for transportation and absorption of nutrients. In addition, the pathways of amino acid metabolism and phenylalanine metabolism were upregulated in the $\mathrm{AD}$ group vs control group in the present study. We speculate that these pathways were correlative with the increase of many proteins in alcoholism patient serum or rodent serum with alcohol treatment, and these proteins included hormones, cytokines and neurotransmitters. For instance, numerous studies showed increased plasma levels of IL-8, IL-10, IL-1 $\beta$, IL-6, and TNF $\alpha$ in alcoholdependent subjects [48, 49], which were inflammation markers in the peripheral cycle. Alcohol and the products of its degradation can induce inflammation via many pathways, and produce some pro-inflammation cytokines to peripheral blood. Thus, genes related to amino acid metabolism were more abundant in the microorganisms of the AD group than the other groups. Several earlier studies in rodents and patients have already shown that acute and chronic alcohol consumption can increase gut permeability $[50,51]$. More recent studies in preclinical animal models and in patients with alcohol abuse have also confirmed these findings [52, 53, 54]. However, the exact molecular mechanisms of increased intestinal permeability remain largely unknown. In the present study, metagenomic prediction at KEGG level 2 showed that membrane transport was the most dominant gene family. Whether it is 
related with increased intestinal permeability needs to be examined further.

In conclusion, this study mainly compared the microbiota community compositions in the jejunum and colon contents in the chronic alcohol-exposed rats. The study demonstrated that chronic alcohol consumption does not significantly alter the microbiota diversity and richness in the jejunum and colon. However, it has profound effects on the microbiota structure in the colon but few effects on the jejunum. Alcohol abuse alters the colonic microbial composition, and disturbs the balance of intestinal microbes, leading to a condition called dysbiosis. Gut dysbiosis may contribute to alcohol use disorders by many pathways, including increasing gut leakiness, the production of proinflammatory cytokines, and LPS. Thus, from the clinical perspective, understanding the effect of alcohol on intestinal microbiota composition, as well as measure for correcting intestinal dysbiosis by modulating the gut bacteria are practicable in treating alcohol-induced disease.

\section{Acknowledgments}

This research was supported by the basic research project of Henan Provincial Science and Technology Department (grant 142300410026) and Science and Technology Research Project of Henan Province (grant 182102310553). The authors express their gratitude to the anonymous reviewers for their valuable comments and suggestions.

\section{Conflict of Interest}

The authors have no financial conflicts of interest to declare.

\section{References}

1. Heilig M, Egli M. 2006. Pharmacological treatment of alcohol dependence: target symptoms and target mechanisms. Pharmacol. Ther. 111: 855-876.

2. Thoma P, Friedmann C, Suchan B. 2013. Empathy and social problem solving in alcohol dependence, mood disorders and selected personality disorders. Neurosci. Biobehav. Rev. 37: 448-470.

3. de Timary P, Leclercq S, Stärkel P, Delzenne N. 2015. A dysbiotic subpopulation of alcohol-dependent subjects. Gut. Microbes 6: 388-391.

4. Rehm J, Dawson D, Frick U, Gmel G, Roerecke M, Shield KD, et al. 2014. Burden of disease associated with alcohol use disorders in the United States. Alcohol Clin. Exp. Res. 38: 1068-1077.
5. Levey DF, Leniculescu H, Frank J, Ayalew M, Jain N, Kirlin B, et al. 2014. Genetic risk prediction and neurobiological understanding of alcoholism. Transl. Psychiatry 4: e391.

6. Salvatore JE, Aliev F, Edwards AC, Evans DM, Macleod J, Hickman M, et al. 2014. Polygenic scores predict alcohol problems in an independent sample and show moderation by the environment. Genes 5: 330-346.

7. Whelan R, Watts R, Orr CA, Althoff RR, Artiges E, Banaschewski T, et al. 2014. Neuropsychosocial profiles of current and future adolescent alcohol misusers. Nature 512: 185-189.

8. Gilpin NW, Koob GF. 2008. Neurobiology of alcohol dependence: focus on motivational mechanisms. Alcohol Res. Health 31: 185-195.

9. Freeman K, Staehle MM, Vadigepalli R, Gonye GE, Ogunnaike BA, Hoek JB, et al. 2013. Coordinated dynamic gene expression changes in the central nucleus of the amygdala during alcohol withdrawal. Alcohol Clin. Exp. Res. 37(suppl): E88-E100.

10. Gorky J, Schwaber J. 2016. The role of the gut-brain axis in alcohol use disorders. Prog. Neuropsychopharmacol. Biol. Psychiatry 4: 234-241.

11. Leclercq S,de Timary P, Delzenne NM, Stärkel P. 2017. The link between inflammation, bugs, the intestine and the brain in alcohol dependence. Transl. Psychiatry 7: e1048.

12. Leo Galland. 2014. The Gut Microbiome and the Brain. J. Med. Food 17: 1-12.

13. Sharon G, Sampson TR, Geschwind DH, Mazmanian SK. 2016. The Central Nervous System and the Gut Microbiome. Cell 167: 915-932.

14. Qin J, Li Y, Cai Z, Li S, Zhu J, Zhang F, et al. 2012. A meta genome-wide association study of gut microbiota in type 2 diabetes. Nature 490: 55-60.

15. Hanski I, von Hertzen L, Fyhrquist N, Koskinen K, Torppa K, Laatikainen T, et al. 2012. Environmental biodiversity, human microbiota, and allergy are interrelated. Proc. Natl. Acad. Sci. USA 109: 8334-8339.

16. Dalal SR, Chang EB. 2014. The microbial basis of inflammatory bowel diseases, J. Clin. Invest. 124: 4190-4196.

17. Jess T. 2014. Microbiota, antibiotics, and obesity. N. Engl. J. Med. 371: 2526-2528.

18. Rogers GB, Keating DJ, Young RL, Wong ML, Licinio J, Wesselingh S. 2016. From gut dysbiosis to altered brain function and mental illness: mechanisms and pathways. Mol. Psychiatry 21: 738-748.

19. Foster JA, McVey Neufeld KA. 2013. Gut-brain axis: how the microbiome influences anxiety and depression. Trends Neurosci 36: 305-312.

20. Sharon G, Sampson TR, Geschwind DH, Mazmanian SK. 2016. The central nervous system and the gut microbiome. Cell 167: 915-932.

21. Vuong HE, Hsiao EY. 2017. Emerging roles for the gut microbiome in autism spectrum disorder. Biol. Psychiatry 81: 
411-423.

22. Cani PD, Everard A. 2015. Harnessing genes and diet to fine-tune the gut microbial fitness. Cell Metab. 22: 754-756.

23. Hollister EB, Gao C, Versalovic J. 2014. Compositional and functional features of the gastrointestinal microbiome and their effects on human health. Gastroenterology 146: 14491458.

24. Mutlu EA, Gillevet PM, Rangwala H, Sikaroodi M, Naqvi A, Engen PA, Kwasny M, et al. 2012. Colonic microbiome is altered in alcoholism. Am. J. Physiol. Gastrointest Liver Physiol. 302: G966-G978.

25. Engen PA, Green SJ, Voigt RM, Forsyth CB, Keshavarzian A. 2015. The gastrointestinal microbiome: alcohol effects on the composition of intestinal microbiota. Alcohol Res. 37: 223-236.

26. Bishehsari F, Magno E, Swanson G, Desai V, Voigt RM, Forsyth $\mathrm{CB}$, et al. 2017. Alcohol and gut-derived inflammation. Alcohol Res. 38: 163-171.

27. Bull-Otterson L, Feng W, Kirpich I, Wang Y, Qin X, Liu Y, et al. 2013. Metagenomic analyses of alcohol induced pathogenic alterations in the intestinal microbiome and the effect of Lactobacillus rhamnosus GG treatment. PLoS One 8: e53028.

28. Zoetendal EG, Rajilic-Stojanovic M, de Vos WM. 2008. Highthroughput diversity and functionality analysis of the gastrointestinal tract microbiota. Gut 57: 1605-1615.

29. Zhao WJ, Wang YP, Liu SY, Huang JJ, Zhai ZX, He C, et al. 2015. The dynamic distribution of porcine microbiota across different ages and gastrointestinal tract segments. PLoS One 10: $\mathrm{e} 0117441$.

30. Erden BF, Ozdemirci S, Yildiran G, Utkan T, Gacar N, Ulak G. 1999. Dextromethorphan attenuates ethanol withdrawal syndrome in rats. Pharmacol. Biochem. Behav. 62: 537-541.

31. Chen Y, Yang F, Lu H, Wang B, Chen Y, Lei D, et al. 2011. Characterization of fecal microbial communities in patients with liver cirrhosis. Hepatology 54: 562-572.

32. Queipo-Ortuño MI, Boto-Ordóñez M, Murri M, GomezZumaquero JM, Clemente-Postigo M, Estruch R, et al. 2012. Influence of red wine polyphenols and ethanol on the gut microbiota ecology and biochemical biomarkers. Am. J. Clin. Nutr. 95: 1323-1334.

33. Mutlu E, Keshavarzian A, Engen P, Forsyth CB, Sikaroodi M, Gillevet P. 2009. Intestinal dysbiosis: a possible mechanism of alcohol-induced endotoxemia and alcoholic steatohepatitis in rats. Alcohol Clin. Exp. Res. 33: 1836-1846.

34. Yan AW, Fouts DE, Brandl J, Stärkel P, Torralba M, Schott E, et al. 2011. Enteric dysbiosis associated with a mouse model of alcoholic liver disease. Hepatology 53: 96-105.

35. Mu C, Yang Y, Su Y, Zoetendal EG, Zhu W. 2017. Differences in microbiota membership along the gastrointestinal tract of piglets and their differential alterations following an earlylife antibiotic intervention. Front. Microbiol. 8: 797.

36. Xiao HW, Ge C, Feng GX, Li Y, Luo D, Dong JL, et al. 2018. Gut microbiota modulates alcohol withdrawal-induced anxiety in mice. Toxicol. Lett. 287: 23-30.

37. Peterson VL, Jury NJ, Cabrera-Rubio R, Draper LA, Crispie F, Cotter PD, et al. 2017. Drunk bugs: chronic vapour alcohol exposure induces marked changes in the gut microbiome in mice. Behav. Brain Res. 323: 172-176.

38. Levitt MD, Li R, DeMaster EG, Elson M, Furne J, Levitt DG. 1997. Use of measurements of ethanol absorption from stomach and intestine to assess human ethanol metabolism. Am. J. Physiol. 273: G951-G957.

39. Norberg A, Jones AW, Hahn RG, Gabrielsson JL. 2003. Role of variability in explaining ethanol pharmacokinetics: research and forensic applications. Clin. Pharmacokinet 42: 1-31.

40. Zakhari S. 2006. Overview: how is alcohol metabolized by the body? Alcohol Res. Health 29: 245-254.

41. Nosova T, Jousimies-Somer $H$, Jokelainen $K$, Heine $R$, Salaspuro M. 2000. Acetaldehyde production and metabolism by human indigenous and probiotic Lactobacillus and Bifidobacterium strains. Alcohol Alcohol 35: 561-568.

42. Leclercq S, Matamoros S, Cani PD, Neyrinck AM, Jamar F, Stärkel P, et al. 2014. Intestinal permeability, gut-bacterial dysbiosis, and behavioral markers of alcohol-dependence severity. Proc. Natl. Acad. Sci. USA 111: E4485-E4493.

43. Leclercq S, De Saeger C, Delzenne N, de Timary P, Stärkel P. 2014. Role of inflammatory pathways, blood mononuclear cells, and gut-derived bacterial products in alcohol dependence. Biol. Psychiatry 76: 725-733.

44. Kirpich IA, Solovieva NV, Leikhter SN, Shidakova NA, Lebedeva OV, Sidorov PI, et al. 2008. Probiotics restore bowel flora and improve liver enzymes in human alcoholinduced liver injury: a pilot study. Alcohol 42: 675-682.

45. Dubinkina VB, Tyakht AV, Odintsova VY, Yarygin KS, Kovarsky BA, Pavlenko AV, et al. 2017. Links of gut microbiota composition with alcohol dependence syndrome and alcoholic liver disease. Microbiome 5: 141.

46. Nimgampalle M, Kuna Y. 2017. Anti-Alzheimer Properties of Probiotic, Lactobacillus plantarum MTCC 1325 in Alzheimer's disease induced Albino Rats. J. Clin. Diagn. Res. 11: KC01-KC05.

47. Wang H, Lee IS, Braun C, Enck P. 2016. Effect of probiotics on central nervous system functions in animals and humans: a systematic review. J. Neurogastroenterol Motil 22: 589-605.

48. Leclercq S, Cani PD, Neyrinck AM, Stärkel P, Jamar F, Mikolajczak M, et al. 2012. Role of intestinal permeability and inflammation in the biological and behavioral control of alcohol-dependent subjects. Brain Behav. Immun. 26: 911-918.

49. Parlesak A, Schäfer C, Schütz T, Bode JC, Bode C. 2000. Increased intestinal permeability to macromolecules and endotoxemia in patients with chronic alcohol abuse in different stages of alcohol-induced liver disease. J. Hepatol. 32: $742-747$.

50. Bode C, Bode JC. 2003. Effect of alcohol consumption on the gut. Best Pract Res. Clin. Gastroenterol 17: 575-592.

51. Keshavarzian A, Farhadi A, Forsyth CB, Rangan J, Jakate S, 
Shaikh M, et al. 2009. Evidence that chronic alcohol exposure promotes intestinal oxidative stress, intestinal hyperpermeability and endotoxemia prior to development of alcoholic steatohepatitis in rats. J. Hepatol 50: 538-547.

52. de Timary P, Stärkel P, Delzenne NM, Leclercq S. 2017. A role for the peripheral immune system in the development of alcohol use disorders? Neuropharmacology 122: 148-160.

53. Donnadieu-Rigole H, Pansu N, Mura T, Pelletier S, Alarcon R,
Gamon L, et al. 2018. Beneficial effect of alcohol withdrawal on gut permeability and microbial translocation in patients with alcohol use disorder. Alcohol. Clin. Exp. Res. 42: 32-40.

54. Stärkel P, Leclercq S, de Timary P, Schnabl B. 2018. Intestinal dysbiosis and permeability: the yin and yang in alcohol dependence and alcoholic liver disease. Clin. Sci. (Lond) 132: 199-212. 